\title{
Trends in the Incidence and Treatment of Cerebrovascular Diseases in Korea : Part I. Intracranial Aneurysm, Intrace- rebral Hemorrhage, and Arteriovenous Malformation
}

\author{
Si Un Lee, ${ }^{1,2}$ Tackeun Kim, ${ }^{1,2}$ O-Ki Kwon, ${ }^{1,2}$ Jae Seung Bang, ${ }^{1,2}$ Seung Pil Ban, ${ }^{1,2}$ Hyoung Soo Byoun, ${ }^{1,2}$ Chang Wan Oh ${ }^{1,2}$ \\ Department of Neurosurgery, Seoul National University Bundang Hospital, Seongnam, Korea \\ Department of Neurosurgery, ${ }^{2}$ Seoul National University College of Medicine, Seoul, Korea
}

Objective : To analyze the incidence and treatment trends of hemorrhagic stroke (HS), according to HS subtypes, using nationwide data in Korea from January 2008 to December 2016.

Methods : We used data from the national health-claim database provided by the National Health Insurance Service for 2008-2016 using the International Classification of Diseases. The crude incidence and age-standardized incidence of each disease associated with HS, which included intracranial aneurysm (IA), hypertensive intracerebral hemorrhage (ICH), and arteriovenous malformation (AVM), were calculated and additional analysis was conducted according to age and sex. Changes in trends in treatment methods and number of treatments were analyzed for each cerebrovascular disease using the Korean Classification of Diseases procedure codes.

Results : In 2016, the total number of newly diagnosed adult patients with HS was 24169 , showing a decrease by $7.0 \%$ since 2008; the age-standardized incidence of HS was 46.2 per 100000 person-years. The age-standardized incidence of unruptured IA (UIA) in adults was 71.4 per 100000 person-years -increased by 2.6-fold since 2008-while that of ruptured IA (RIA) was 12.6 per 100000 person-years, which had decreased at a rate of $20.3 \%$ since 2008. The number of coil embolization (CE) for UIA increased by 3.4-fold over 9 years and exceeded that of clipping since 2008. With respect to RIA, CE increased by 2.0-fold over 9 years and exceeded that of clipping from 2014. As for spontaneous ICH in adults, the age-standardized incidence was 31.3 per 100000 personyears in 2016 - decreased by 34.7\% since 2008 - and 14.6\% of patients diagnosed with ICH were treated in 2016, which was not significantly different from the proportion of patients treated since 2008. The age-standardized incidence of unruptured AVM (UAVM) was 2.0 per 100000 person-year in 2016, while that of ruptured AVM (RAVM) was 2.4 per 100000 person-years in 2016, showing a decreasing rate of 17.2\% from 2008. The total number of treated patients with AVM declined since 2014.

Conclusion : In Korea, age-related cerebral vascular diseases, such as RIA, ICH, and RAVM, demonstrated a declining trend in agestandardized incidence; meanwhile, UIA and UAVM demonstrated an increased trend in both crude incidence and age-standardized incidence for 9 years. The increase in the elderly population, management of hypertension, and development of diagnostic and endovascular techniques appear to have influenced this trend.

Key Words : National health service · Intracranial aneurysm · Cerebral hemorrhage · Arteriovenous malformation · Incidence.

- Received : September 4, 2018 •Accepted : November 11, 2018

- Address for reprints : Chang Wan Oh

Department of Neurosurgery, Seoul National University Bundang Hospital, 82 Gumi-ro 173beon-gil, Bundang-gu, Seongnam 13620, Korea

Tel : +82-31-787-7169, Fax : +82-31-787-4059, E-mail : wanogh@snubh.org, ORCID : https://orcid.org/0000-0001-7311-7072

This is an Open Access article distributed under the terms of the Creative Commons Attribution Non-Commercial License (http://creativecommons.org/licenses/by-nc/4.0) which permits unrestricted non-commercial use, distribution, and reproduction in any medium, provided the original work is properly cited. 


\section{INTRODUCTION}

Cerebrovascular diseases (CVDs), including ischemic stroke (IS) and hemorrhagic stroke (HS), are the third most common cause of death worldwide, with a mortality rate of 86.5 per 100000 person-years (2016; IS, 43.4 per 100000 person-years; HS, 43.1 per 100000 person-years $)^{11}$. Although mortality rates from IS and HS, combined, in developed countries, were almost halved from 1990 to 2013, the absolute number of people affected, globally, by both IS and HS, over that time period, has significantly increased ${ }^{17)}$. Moreover, as the population ages and accessibility to imaging tests increases, the detection rate and incidence of CVD has increased annually ${ }^{16,19,31,32)}$, and the guidelines for treatment is changing accordingly ${ }^{1,14,30)}$. Although nationwide population-based studies on the epidemiology of cerebral stroke have been reported, few studies have analyzed the subtype of hemorrhagic stroke, despite a high proportion of HS (30.3-35.1\%) accounting for overall stoke.

The National Health Insurance Service (NHIS) in Korea is a nationwide universal insurance system that is responsible for the nation's health care and medical bills; thus, this database includes hospital records for $97 \%$ of the Korean population. Furthermore, the NHIS operates a severe disease registration system, which supports medical expenses for patients, and for which strict diagnostic criteria must be met for registration. Accordingly, the NHIS database, which covers a single-ethnicity population of 50 million people, is optimal for the epidemiological study of HS.

Therefore, we analyzed the incidence and treatment trends of hemorrhagic stroke, according to subtypes, using NHIS data from January 2005 to December 2016.

\section{MATERIALS AND METHODS}

\section{Data source and cohort design}

We used data from the national health-claim database provided by the NHIS for 2005-2016. Patients that had been diagnosed with CVD during the preceding 3 years at any clinic or hospital were excluded from selected incident cases, which were different from prevalent cases. To secure a 3-year washout period, we used 2008 as the index year; thus, the total observation period of the reconstructed cohort was 9 years (2008-2016). We extracted only data from tertiary referral general hospitals (TRGH), general hospitals (GH), semi-hospitals (SH), and primary medical institutions to increase confidence in medical information. We excluded other medical institutions where medical information was not clear. This study was approved by Institutional Review Board at Seoul National University Bundang Hospital (X-1703-388-909), which waived informed consent.

Using the International Classification of Diseases, 10th Revision (ICD-10) diagnostic codes, we collected data from patients with CVD associated with hemorrhagic stroke, which included intracranial aneurysm (IA), hypertensive intracerebral hemorrhage (ICH), and arteriovenous malformation (AVM). Patients must have been diagnosed for the first between January 2008 and December 2016.

\section{Study population}

\section{Intracranial aneurysm}

We identified patients with unruptured IA (UIA) in each year using the I67.1 ICD code. Patients in whom a UIA subarachnoid hemorrhage (SAH) had been diagnosed within 3 years prior to the index period or patients who reported any brain hemorrhage (e.g., I60, I61, and I62) or history of brain trauma during the index period were excluded.

Patients with ruptured IA (RIA), diagnosed for the first time during 2008-2016, were identified using the I60 ICD codes, excluding I60.8 (meningeal hemorrhage and rupture of cerebral arteriovenous malformation). We included only patients admitted with $\geq 7$ days of hospitalization, or patients who died within 14 days of hospitalization, and excluded patients who were hospitalized $<7$ days so as not to overestimate the number of patients with aneurysm ruptures, as patients with ruptures are usually hospitalized for $>7$ days to manage vasospasm in South Korea. In addition, patients in whom a SAH had been diagnosed within 3 years prior to the index period or who had history of brain trauma during the index period were also excluded.

Regarding patients diagnosed with UIA or RIA, this study included only patients for whom the use of diagnostic modalities such as computed tomography angiography (CTA), magnetic resonance angiography (MRA), or digital subtraction angiography (DSA) within 4 weeks from the initial diagnosis were reported. We also excluded pediatric patients younger than 20 years because the pathophysiology of pediatric IA is 
different from that of adult $\mathrm{IA}^{10,22,24)}$.

\section{Intracerebral hemorrhage}

Patients diagnosed with ICH were identified using the I61 ICD code. We included only patients admitted with $\geq 3$ days of hospitalization or patients who died within 3 days of hospitalization, with the same ICD-10 code, to avoid including patients with old hemorrhages. In addition, patients with a history of CVD, diagnosed within 3 years prior to the index period, and patients with other causes of brain injury (e.g., brain trauma and brain tumor), were excluded. We excluded patients with IA, AVM, and cavernous malformation (CM); thus, only patients diagnosed with hypertensive ICH were included. When assessing intracerebral hemorrhage, we also excluded pediatric patients younger than 20 years because spontaneous childhood ICH is due mostly to vascular malformations ${ }^{4)}$.

\section{Arteriovenous malformation}

We identified patients with unruptured AVM using the Q28.2 ICD code omitting I60, I61, I62, Q28.3, or D18.01, to exclude ruptured AVM or CM. Additionally, we recruited only patients for whom the use of diagnostic modalities such as CTA, MRA, or DSA within 4 weeks from the initial diagnosis were performed. This criterion was also applied to ruptured AVM. Patients in whom an AVM or CM had been diagnosed within 3 years prior to the index period were excluded.

Patients diagnosed with ruptured AVM were identified using the I60.8 ICD code or Q28.2 with I60 or I61, which indicated intracranial hemorrhage and excluded Q28.3 or D18.01, which are the ICD codes for CM. We included only patients admitted with $\geq 3$ days of hospitalization or patients who died within 3 days of hospitalization, with the same ICD-10 code, to avoid including patients with a previously ruptured AVM. Additionally, patients in whom a ruptured AVM or CM had been diagnosed within 3 years prior to the index period were excluded.

\section{Data analysis}

\section{Incidence of cerebrovascular diseases}

We performed data manipulation and extraction using SAS version 9.4 (SAS Institute Ins., Cary, NC, USA). The annual crude incidence was calculated for each CVD instead of overall crude incidence because the incidence of CVD changed annually. First, the number of cases of CVD, diagnosed for the first time from 2008 to 2016, was obtained. Then, the annual crude incidence of CVD was calculated using the total population of each year, which had been corrected for the number of deaths and births in each year. In addition, the annual crude incidence of each CVD according to age and sex were also obtained. The standardized incidence of each CVD was deduced from the crude incidence using the formula below, after correcting for demographic bias in this cohort relative to the national population structure $\left(\mathrm{P}_{\mathrm{i}}=\right.$ proportion of each age / sex group of the national population; $\mathrm{I}_{\mathrm{i}}=$ incidence of each age / sex group of the cohort population).

Annual crude incidence $=\frac{\text { Number of case } \times 100000}{\text { Total observation size }(\text { person } \times 1 \text { year })}$

Standardized incidence $=\sum_{i} P_{i} * I_{i}$

In addition, the age-specific incidence was calculated to obtain the crude incidence for each age group, with the agegroups being defined in 10 -year periods.

\section{Treatment for cerebrovascular diseases}

Change in trend of treatment method and number of treatments were analyzed for each CVD, and change in number of treatments by region and hospital size were also analyzed. Treatments for UIA or RIA consisted of surgical clipping, defined using procedure codes S4641 and S4642 in the Korean Classification of Diseases (KCD) procedure codes, and endovascular coiling, defined using codes M1661 and M1662. As for the treatment of $\mathrm{ICH}$, stereotactic aspiration was defined using codes N0323 and S4755 and KCD codes S4622 and N0333, for surgeries with craniotomy or craniectomy. Craniotomy for AVM was coded as S4652-S4654 and AVM embolization was defined with codes M1663-M1666. Finally, stereotactic radiosurgery (SRS) was defined using codes HD113 and HD114.

\section{Statistical analysis}

Statistics were performed using SPSS version 22 (IBM Corp., Armonk, NY, USA). Spearman's rank-order correlation was used to determine the linear relationship of trend with $p$ values and Spearman's coefficient (r). The Wilcoxon signed rank test was used to confirm the change in the variables each year. A $p$-value $<0.05$ was regarded as statistically significant. 

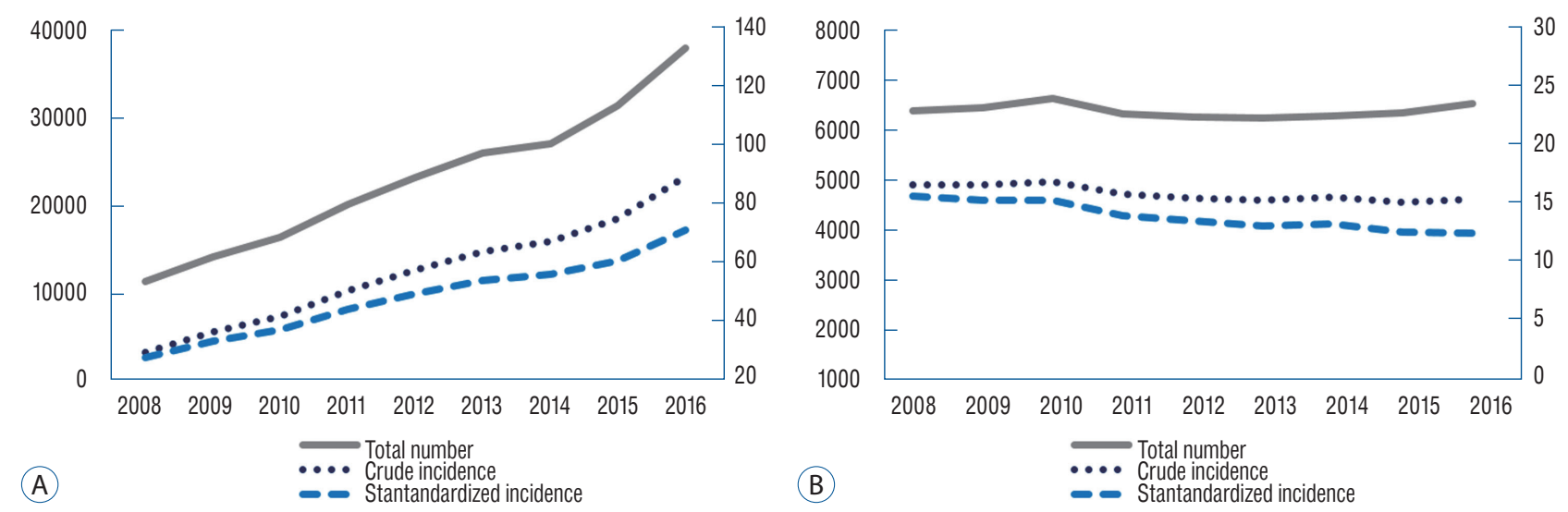

Fig. 1. A : Total number of patients diagnosed with UIA and both the crude incidence and age-standardized incidence of UIA showed an increasing trend over 9 years. B : Total number of patients diagnosed with RIA and the crude incidence revealed no significant change from 2008 to 2016. Meanwhile, the age-standardized incidence of RIA steadily decreased over the 9 examined years. UIA : unruptured intracranial aneurysm, RIA : ruptured intracranial aneurysm.
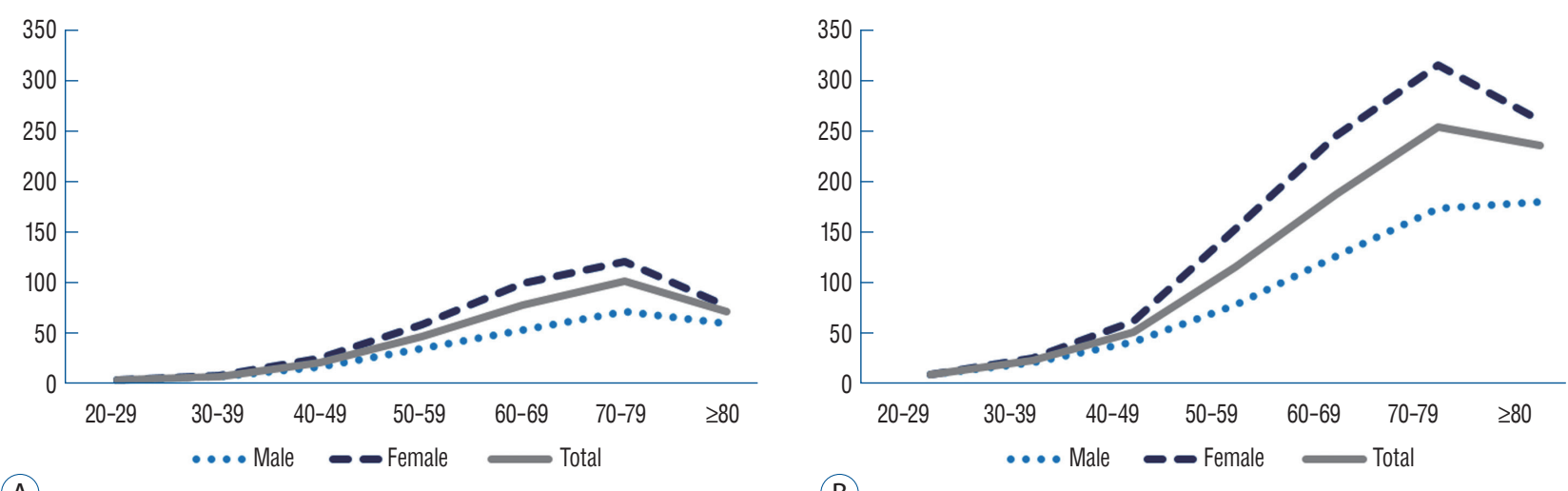

(A)
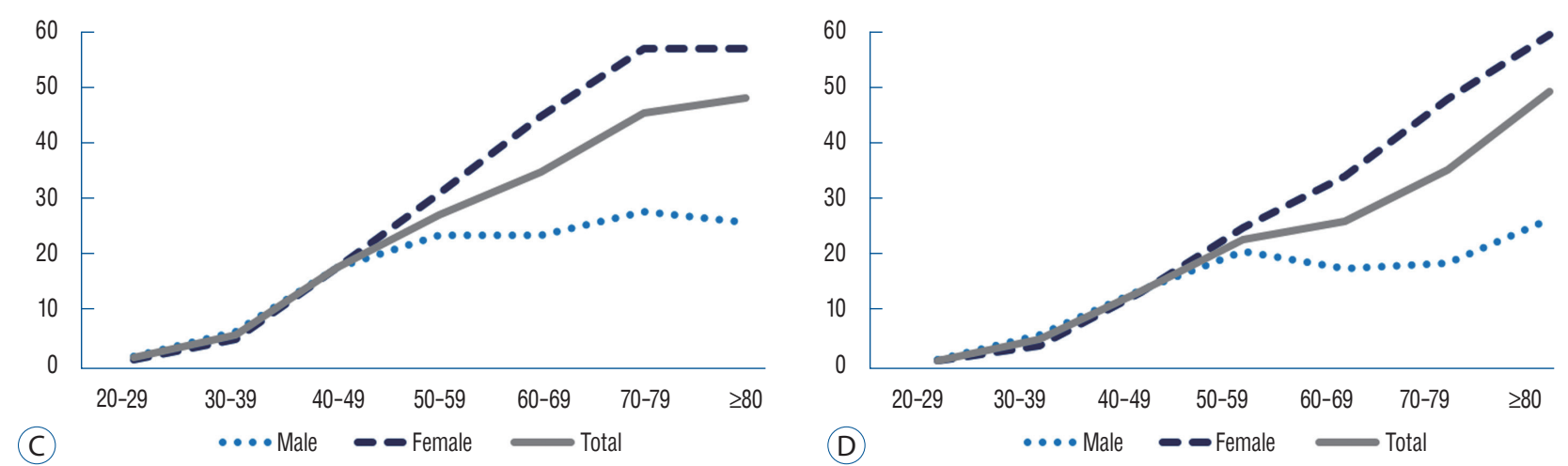

Fig. 2. A and B : Crude incidence for each age group diagnosed with UIA in 2008 (A) and 2016 (B). The age-specific incidence increased for all ages over 9 years and was highest for patients in their 70s. In patients in their $20 \mathrm{~s}$ or older, the incidence in female patients was higher than the incidence in male patients; in 2016, the largest difference was seen in patients in their 50s; the incidence in female patients was 2.0-times greater than that in male patients. C and D : Age-specific incidence for each age group diagnosed with RIA in 2008 (C) and 2016 (D). For all ages except those in their 80 s, the agespecific incidence decreased over 9 years. In patients in their 50 s or older, the incidence in female patients exceed that in male patients. The difference in incidence according to sex tends to increase with age; thus, in 2016, the incidence in female patients in their 60s and 70s was 1.9-times and 2.6-times higher than that in male patients, respectively. UIA : unruptured intracranial aneurysm, RIA : ruptured intracranial aneurysm. 


\section{RESULTS}

\section{Intracranial aneurysm}

\section{Incidence of intracranial aneurysm}

The number of adult patients diagnosed with UIA in 2008 and 2016 were 11256 and 37997, respectively - an annual increasing rate of $29.7 \%$. In 2016, both the crude incidence and age-standardized incidence were shown to have increased steadily ( $p<0.01, \mathrm{r}=1.0)$ and were calculated as 90.0 per 100000 person-year and 71.4 per 100000 person-years, respectively (Fig. 1A). The number of patients diagnosed with RIA for the first time in 2008 and 2016 was 6389 and 6543, respectively, with a crude incidence of 16.8 per 100000 person-years and 15.5 per 100000 person-years, respectively. This finding demonstrated a significant decline over the 9 years $(p<0.01, r=-0.83)$. In addition, the age-standardized incidence of RIA was 12.6 per
100000 person-years in 2016, which was decreased from 2008 at a rate of $20.3 \%(p<0.01, \mathrm{r}=-0.97)$ (Fig. 1B).

Overall, the incidence of UIA increased annually for all ages, and in 2016, the number of cases diagnosed among patients aged 60 years exceeded the number of cases diagnosed among patients aged 50 years. The age-specific incidence for each age group was the highest for 70 years, and the age-specific incidence for patients older than 50 years exceeded the overall crude incidence (Fig. 2A and B). Regarding RIAs, the number of cases with a diagnosed RIA was the highest in 50 years and the age-specific incidence for patients older than 50 years exceeded the overall crude incidence in 2016. For UIA and RIA, the rate of diagnosis in patients older than 60 years was $56.5 \%$ and $47.3 \%$, respectively, in 2016 - an increase from $52.6 \%$ and $42.1 \%$ in 2008, respectively (Fig. 2C and D).

Regarding the crude incidence according to sex, the crude incidence of UIA in female patients exceeded that of male pa-

(A)
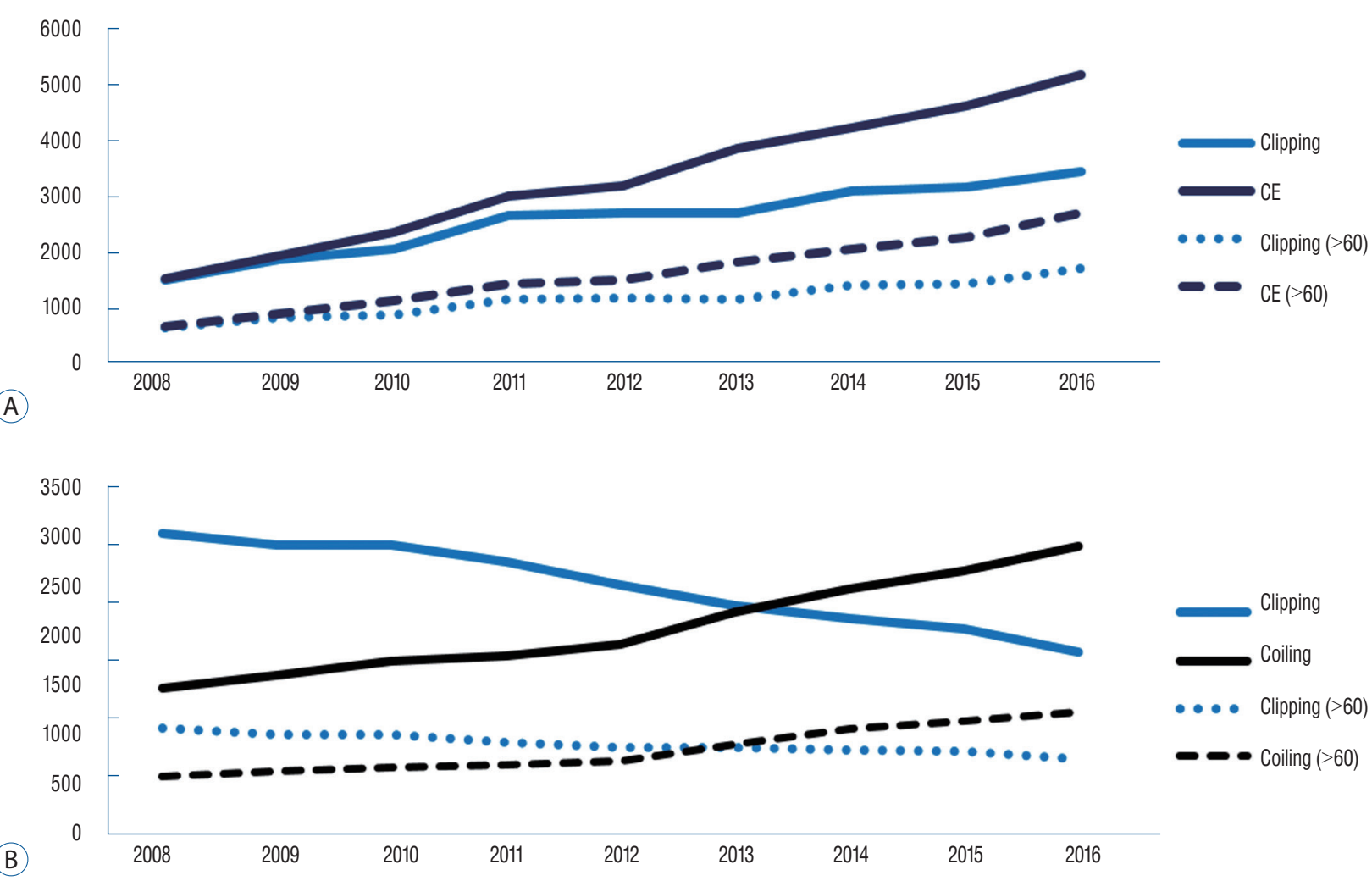

Fig. 3. A : In the case of UIA, CE increased by $29.5 \%$ per year over 9 years and clipping increased by $16.0 \%$ per year. The percentage of patients older than 60 years who were treated with clipping and CE treatment increased from $44.3 \%$ and $44.6 \%$ in 2008 to $50.1 \%$ and $52.6 \%$ in 2016 , respectively. B : For RIA, CE increased by $97.4 \%$ over 9 years, but clipping decreased by $39.2 \%$. CE has exceeded clipping since 2014 and was reported 2.0 -times more often than clipping in 2016. The number of CEs performed in patients older than 60 years increased by $114.5 \%$, but the number of clipping cases decreased by $28.5 \%$. Overall, the number of treated patients older than 60 years increased from $36.6 \%$ of total treated patients for RIA in 2008 to $42.2 \%$ in 2016. UIA : unruptured intracranial aneurysm, CE : coil embolization, RIA : ruptured intracranial aneurysm. 
tients in their 20s and was 1.9-times higher than in male patients in their 60s in 2016. Regarding RIAs, the crude incidence in female patients exceeded that in male patients in their 50s and was 2.6-times higher than in male patients in their 70s in 2016 (Fig. 2).

\section{Treatment for intracranial aneurysm}

The number of patients who underwent coil embolization (CE) for UIA in 2016 was 5162. This number increased 3.4fold, with an increasing annual rate of $29.5 \%$, over 9 years $(p<0.01, r=0.98)$. The number of patients who underwent clipping in 2016 was 3446. This number increased 2.3-fold, with an increasing annual rate of $16 \%$, over 9 years $(p<0.01, r=1.0)$. CE has exceeded clipping since 2008 and was reported 1.5-fold more frequently than clipping in 2016 (Fig. 3A).

Regarding RIAs, in 2016, $74.6 \%$ of patients, diagnosed with
RIAs for the first time, were treated; 61.2\% underwent CE and $38.8 \%$ underwent clipping. These numbers were comparable with those reported in 2008 when $72.5 \%$ of patients with RIA were treated (CE, 32.7\%; clipping, 67.3\%). The number of patients who underwent CE in 2016 was 2989. This number increased 2.0-fold, with an increasing annual rate of 12.2\%, over 9 years $(p<0.01, \mathrm{r}=1.0)$. In contrast to unruptured aneurysms, the number of patients who underwent clipping decreased with a decreasing annual rate of $4.9 \%(p<0.01, r=-1.0)$. CE has exceeded clipping since 2014 and was 1.6-times more popular than clipping in 2016 (Fig. 3B).

The number of patients older than 60 years who underwent CE for UIA increased 4-fold over 9 years; in 2016, 52.6\% of all patients who underwent CE for UIA were aged over 60 years. In contrast, the number of patients older than 60 years who underwent clipping for UIA increased 2.6-fold over 9 years; in

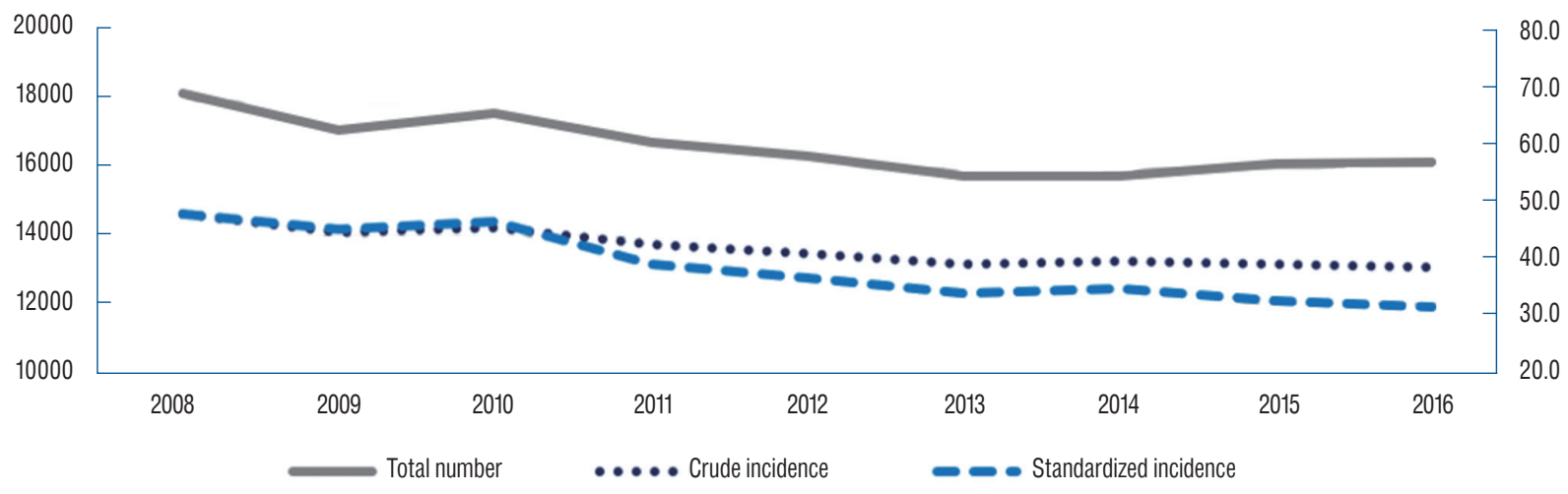

A
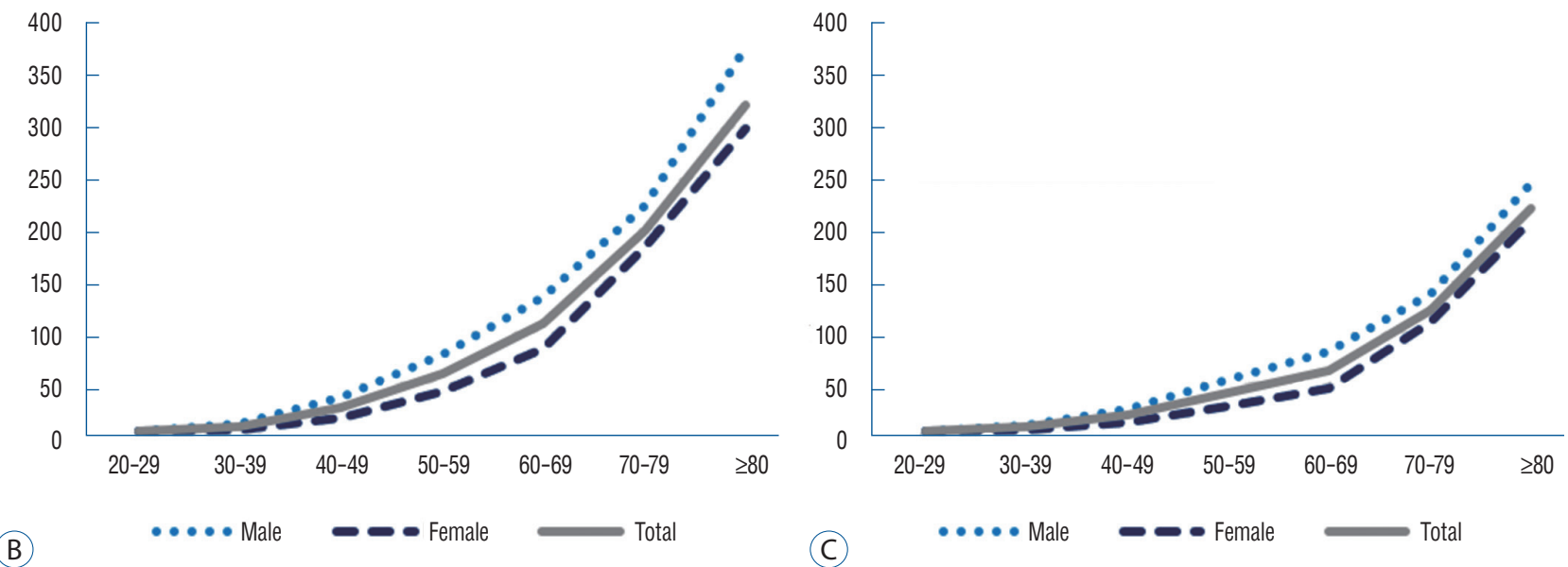

Fig. 4. A : Both the crude incidence and age-standardized incidence of $\mathrm{ICH}$ have declined over the past 8 years, while the decrease in age-standardized incidence is greater. B and C: Age-specific incidence for each age group diagnosed with ICH in 2008 (A) and 2016 (B). Overall, the crude incidence was higher in male patients, and decreased in all ages over 9 years. ICH : intracerebral hemorrhage. 
2016, 50.1\% of all patients who underwent clipping for UIA were over 60 years. Concerning RIAs, $66.6 \%$ of elderly patients diagnosed with RIA were treated in 2016 - an increase from $63.0 \%$ in 2008 . In 2016, 61.8\% of elderly patients treated for RIA underwent CE, while clipping was performed in $382 \%$ of these patients. In 2008, CE was performed in $35.0 \%$ of patients and clipping was performed in $65.0 \%$ of patients (Fig. 3).

\section{Intracerebral hemorrhage}

\section{Incidence of intracerebral hemorrhage}

The number of adult patients diagnosed with ICH in 2008 and 2016 were 18211 and 16174, respectively-a significantly decreasing trend $(p<0.01, \mathrm{r}=-0.82)$. In 2016, the crude incidence and age-standardized incidence were calculated as 38.5 per 100000 person-years and 31.3 per 100000 person-years, respectively-decreased from 48.0 per 100000 person-year in 2008. Age-standardized incidence revealed a significantly decreasing trend even greater than that of crude incidence $(p<0.01, \mathrm{r}=-0.97)$ (Fig. 4A).

While the number of patients in their 80 s diagnosed with ICH increased by $26.6 \%(p<0.01, r=-0.87)$, those in other age groups showed a decreasing trend over 9 years. Whereas, the age-specific incidence in all ages revealed a significantly decreasing trend over 9 years and the age-specific incidence for patients older than 50 years exceeded the overall crude inci- dence. The ratio of patients older than 60 years among the patients diagnosed with ICH was $64.5 \%$ in 2016 , which was not significantly different from 2008 ( $p=0.21, \mathrm{r}=0.47$ ). The crude incidence in male patients was significantly higher compared with that in female patients ( $p=0.018$ ) (Fig. $4 \mathrm{~B}$ and C).

\section{Treatment for intracerebral hemorrhage}

The ratio of patients treated for ICH did not significantly differ over 9 years $(p=0.97, \mathrm{r}=0.02)$ and ranged from $14.5 \%$ to $17.5 \%$. The methods of operation were divided into craniotomy and stereotactic aspiration, and the number of stereotactic aspirations was higher than that of the craniotomies at $57.1 \%$ to $60.2 \%$ per year. The proportion of patients older than 80 years among all treated patients steadily increased from $6.1 \%$ in 2008 to $10.6 \%$ in $2016(p<0.01, \mathrm{r}=0.90)$.

\section{Arteriovenous malformation}

\section{Incidence of arteriovenous malformation}

Although there was no significant linear trend over 9 years, the number of patients diagnosed with unruptured AVM (UAVM) increased by 21.7\% (925 in 2008 to 1126 in 2016) $(p<0.01, \mathrm{r}=0.90)$ and the crude incidence and age-standardized incidence were 2.2 per person-year and 2.0 per 100000 personyears in 2016, respectively (Fig. 5A). Meanwhile, the age-standardized incidence of ruptured AVM (RAVM) showed a decreasing trend from 2.9 person-years in 2008 to 2.4 per 100000 person-
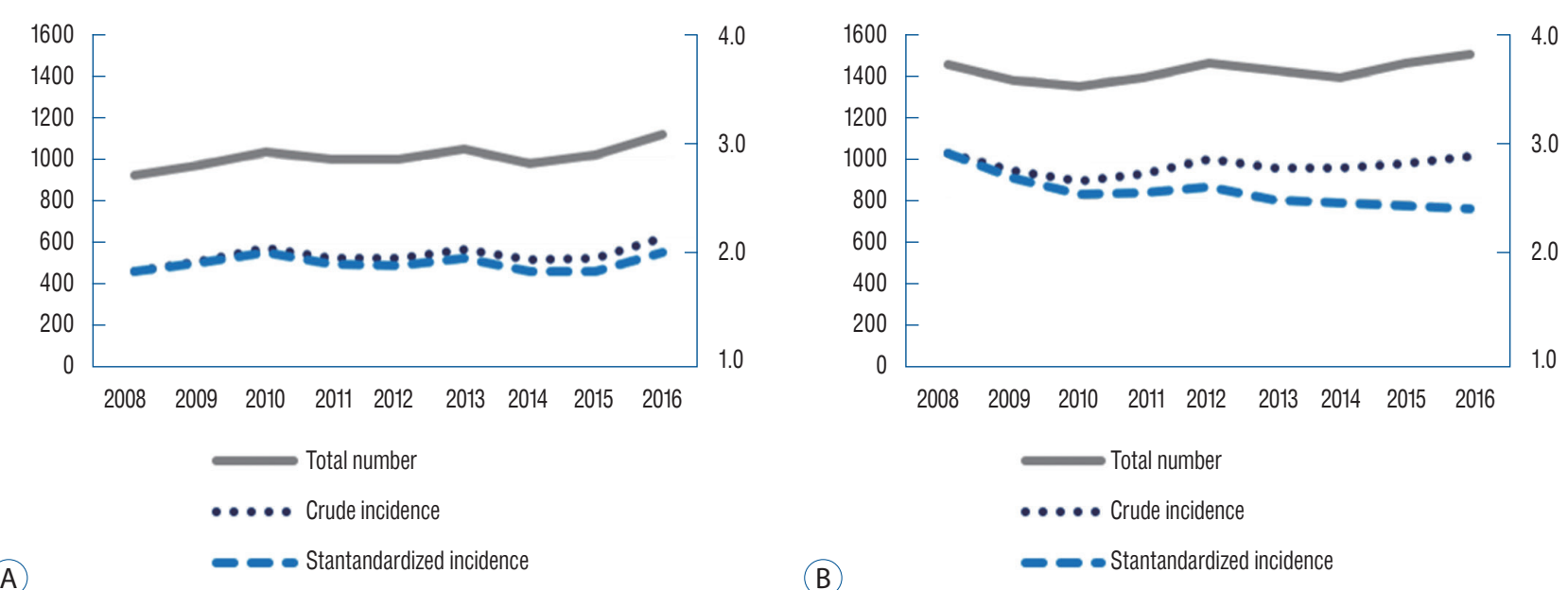

Fig. 5. A : The total number of patients diagnosed with UAVM and both the crude incidence and age-standardized incidence of UAVM showed slight increase over 9 years, although there was no significant linear trend. B : The total number of patients diagnosed with RAVM and the crude incidence revealed no significant change from 2008 to 2016. Meanwhile, the age-standardized incidence of RAVM has been decreasing steadily for 9 years. UAVM : unruptured arteriovenous malformation, RAVM : ruptured arteriovenous malformation. 
years in 2016 ( $p<0.01, \mathrm{r}=-0.93)$, despite the lack of significant change in the crude incidence over 9 years $(p=0.05, \mathrm{r}=0.67)$ (Fig. 5B).

While the number of patients diagnosed with UAVM was highest among patients in their 40s in 2008, the number of patients older than 50 years showed the highest proportion in 2016, with a decrease in the proportion of patients under 50 years and a rise in the proportion in the patients older than 50 years. The age-specific incidence for each age group was the highest in patients aged 60 years, and the age-specific incidence for patients aged over 50 years exceeded the overall crude incidence. Although the number of patients older than 60 years diagnosed with UAVM increased annually $(p<0.01$, $\mathrm{r}=-0.93$ ), it accounted for only $27.4 \%$ of all patients diagnosed with UAVM in 2016 (Fig. 6A and B). The number of patients diagnosed with RAVM was highest for patients in their 50s, whereas the crude incidence of RAVM increased with age.
The proportion of patients aged over 60 years diagnosed was $50.7 \%$ in 2016 , which was increased from $42.4 \%$ in 2008 . Regarding the crude incidence according to sex, female patients older than 60 years tended to have a higher crude incidence than male patients older than 60 years, although the crude incidence of RAVM was not significantly different according to $\operatorname{sex}(p=0.86)$ (Fig. 6C and D).

\section{Treatment for arteriovenous malformation}

Overall, $23.2 \%$ to $28.7 \%$ of patients diagnosed with AVM were treated annually. The rate of treatment increased until 2014, and then decreased to $23.2 \%$ in 2016 . Craniotomy treatment has been declining since 2008 , and only $3.5 \%$ of patients diagnosed with AVM underwent craniotomy in 2016. Embolization and SRS increased until 2014 and 2012, respectively, and then declined to $6.1 \%$ and $11.0 \%$, respectively, in 2016 . Among the patients who underwent gamma knife surgery
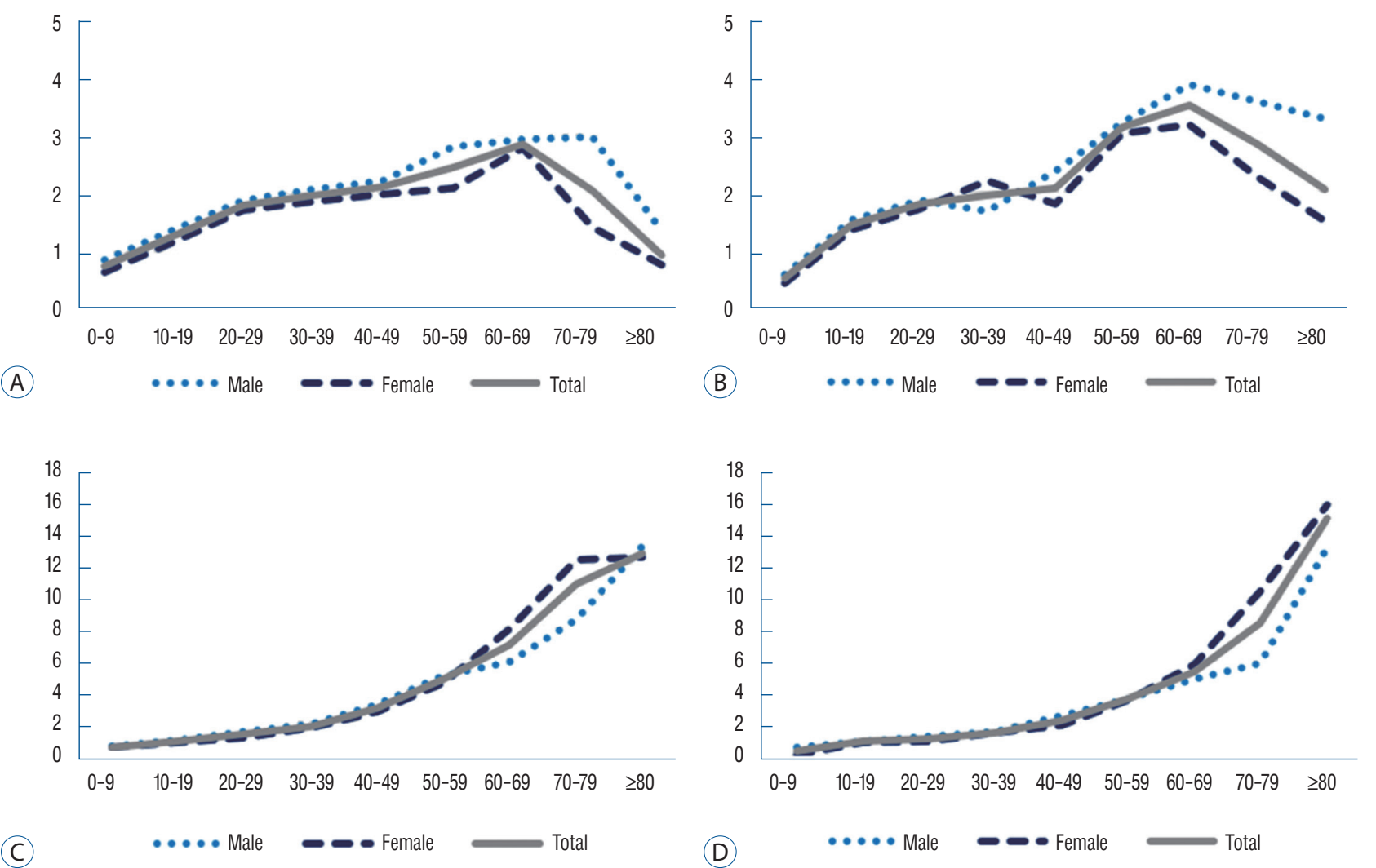

Fig. 6. A and B : Age-specific incidence for each age group diagnosed with UAVM in 2008 (A) and 2016 (B). The age-specific incidence was the highest in 60s and increased in patients older than 50 years over 9 years. Regarding the crude incidence according to sex, female patients over the age of 60 tended to have a higher crude incidence than male patients. C and D: Age-specific incidence for each age group diagnosed with RAVM in 2008 (C) and 2016 (D). The age-specific incidence of RAVM increased with age and decreased in most age groups over 9 years, except in patients older than 80 years, and did not show significant differences according to sex. UAVM: unruptured arteriovenous malformation, RAVM : ruptured arteriovenous malformation. 
(GKS), the proportion of patients who underwent embolization was the highest at $19.6 \%$ in 2014 , but then decreased to $14.4 \%$ in 2016 (Fig. 7).

\section{DISCUSSION}

\section{Intracranial aneurysm}

The incidence of IA has been previously reported in a study based on NHIS data from the National Evidence-based Healthcare Collaborating Agency in Korea. However, the applicability of these findings is limited because only data from 2006 to 2009 were included, although the findings of a previous study based on data from 2008 and 2009 were in line with ours, showing that there is continuity ${ }^{20)}$. According to the results of this study, the crude incidence of UIA increased steadily from 29.6 per 100000 person-years in 2008 to 90.0 per 100000 person-years in 2016. This is probably due to the increased diagnosis rate with increasing number of hospitals performing DSA, magnetic resonance angiography, and computer tomography angiography. It is also possible that the population aged older than 60 years, who have high incidence of UIA, increased by $41.8 \%$ in 2016 compared to 2008 . There- fore, it could be more accurate to define the incidence of UIA as the detection rate rather than the incidence of disease. On the other hand, in cases of RIA, both the crude incidence and age-standardized incidence significantly decreased from 2008, but the decline in the age-standardized incidence was greater than that of the crude incidence. This suggests that the actual incidence is decreasing when the effects of aging are corrected. This may be due to an increase in the treatment of UIA and the management of hypertension through health screening ${ }^{21)}$.

The reason for the increase in the number of treatments for UIA is the increase in the condition's detection, but the increase in hospitals capable of embolization is also an important factor. According to current data from the NHIS, the number of $\mathrm{GH}$ available to perform CE for UIA has increased 2.1-fold and the number of patients who underwent CE in GHs has increased 3.9-fold over 9 years. Meanwhile, the number of patients who underwent CE in TRGHs has increased 3.1-fold, although there was no significant change in the number of TRGHs available to perform CE. With respect to RIAs, the number of patients who underwent CE in GHs and TRGHs has increased 2.2-fold and 1.5-fold over 9 years, respectively.

The increased accessibility and the development of CE in-

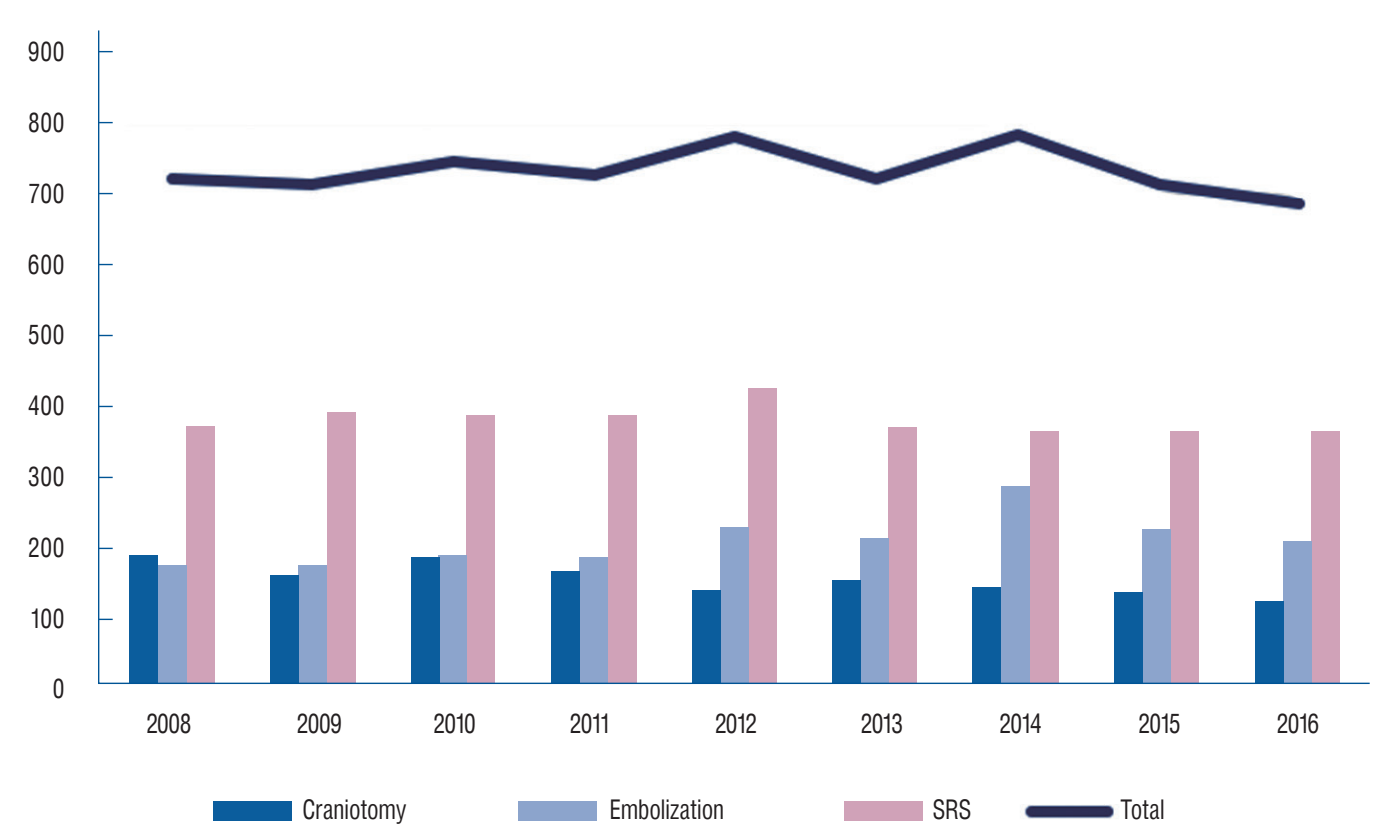

Fig. 7. Craniotomy has been declining since 2008 , and only $3.5 \%$ of patients diagnosed with AVM underwent craniotomy in 2016. Embolization and SRS use increased to $11.9 \%$ in 2014 and $15.9 \%$ in 2012, respectively, and then declined to $6.1 \%$ and $11 \%$, respectively, in 2016 . SRS : stereotactic radiosurgery, AVM : arteriovenous malformation. 
creased the treatment rate of IA in patients aged older than 60 years. The number of patients older than 60 years who were diagnosed with UIA increased by $190.1 \%$ over 9 years, while the number of embolization cases increased by $297.8 \%$. In addition, the rate of treatment for patients diagnosed with RIA older than 60 years increased from $66.6 \%$ in 2008 to $63.0 \%$ in 2016. This is because clipping for RIA in patients aged older than 60 years decreased by $28.5 \%$, while CE for RIA in patients older than 60 years increased by $114.5 \%$.

\section{Intracerebral hemorrhage}

Estimates of ICH incidence around the world vary, but have generally ranged from 10 to 20 per 100000 per years ${ }^{33)}$. Chinese populations generally have a higher proportion of $\mathrm{ICH}$ among all types of stroke than Caucasian populations (17$51 \%$ vs. $6-20 \%$, respectively ${ }^{36}$. In this study, the proportion of ICH among all types of stroke was $16.3 \%$ in 2016, and the age-standardized incidence was 24.3 per 100000 person-years. The proportion of patients older than 80 years increased from $13.5 \%$ in 2008 to $19.3 \%$ in 2016 , while the number of newly diagnosed patients older than 80 years increased by $26.6 \%$. However, the crude incidence in patients older than 80 years decreased by $30.9 \%$ over 9 years. This means that the change in population structure due to the increase in the elderly population affected the incidence of $\mathrm{ICH}$, and it is suggested that health management, such as the diagnosis of hypertension and control of blood pressure, through national health screening is one of the important factors for the decreasing age-standardized incidence ${ }^{21)}$.

According to this study, the proportion of patients treated for ICH ranged from $14.5 \%$ to $17.5 \%$ and stereotactic aspiration was slightly superior to conventional craniotomy, in Korea. As a guideline for the treatment of $\mathrm{ICH}$, the usefulness of craniotomy for supratentorial ICH is not well established because randomized clinical trials (RCTs) demonstrated no overall benefit from early surgery ${ }^{25,26)}$. However, a recent metaanalysis reported that minimally invasive surgery is superior to conventional craniotomy ${ }^{38)}$ and other RCTs showed that minimally invasive surgery with recombinant tissue type plasminogen activator (tPA) is effective for reduction of perihematoma edema with a trend toward improved outcomes ${ }^{26)}$. Furthermore, because a randomized phase 3 clinical trial on minimally invasive surgery plus tPA is currently underway ${ }^{12)}$, there is a possibility that the trends in the treatment of $\mathrm{ICH}$ will change.

\section{Arteriovenous malformation}

The incidence of AVMs according to population-based studies varied from 0.69 to 1.32 per 100000 person-years and the incidence of hemorrhage from AVM is 0.51 to 1.11 per 100000 person-year ${ }^{2,3,5,8,18,27)}$. In our cohort, the age-standardized incidence of UAVM and RAVM were 2.0 per 100000 person-years and 2.4 per 100000 person-years, respectively, which was higher compared with other population-based studies $^{2,3,5,8,18,27)}$. Previous studies identified racial differences in hemorrhagic presentations of AVMs, with non-Caucasian populations demonstrating greater than threefold odds ratio compared with Caucasian populations, which supports the higher incidence found in our cohort ${ }^{15,39)}$.

AVMs were treated in 26.8\% of all AVM patients in 2008 and $28.4 \%$ of these patients in 2014 . However, this percentage declined thereafter, and in 2016 , only $23.2 \%$ of patients were treated. This seems to be related to the ARUBA (A Randomized Trial of Unruptured Brain Arteriovenous Malformations) study, which was published in 2014. The ARUBA trial showed that medical management alone is superior to medical management with interventional therapy for the prevention of death or stroke in patients with unruptured brain arteriovenous malformations followed up for 33 months. However, as the limitations of the ARUBA study have been mentioned, favorable outcomes of microsurgery, GKS, and combined treatments with endovascular embolization have been reported in subsequent studies on ARUBA-eligible patients ${ }^{9,29,35,37)}$. It is also expected that trends in treatment will change as postARUBA studies, such as the BARBADOS study ${ }^{34)}$ and TOBAS study $^{23)}$, proceed.

\section{Limitations}

The strength of the current study is that it analyzed nationwide data from a single-ethnicity population of 50 million people to evaluate trends in the incidence and treatment of CVD associated with HS. However, the study also has limitations. First, we did not calculate the overall crude incidence for each CVD during a sufficient observational period, and thus the understanding of the natural history of each disease is limited. However, the annual crude incidence is considered more appropriate for identifying the trends reflecting the medical environment. Furthermore, it is thought that there will be limitations in obtaining an accurate 
crude incidence for diseases such as CVD, in which the incidence changes rapidly every year, even if a sufficient observational period is considered. Second, inaccuracies in claims data, such as coding inaccuracies, were another limitation of this study. However, a previous study, which validated the diagnosis of CVD based on ICD-10 codes, comparing Korean claims data with medical record reviews, reported a positive predictive value (PPV) of $83.4 \%{ }^{28}$. Additionally, several studies in Taiwan validated national claims data for CVD with ICD codes, revealing high PPVs $(79.0-94.0 \%)^{6,713)}$. Therefore, this study is sufficiently reliable, but these limitations must be considered in the interpretation.

\section{CONCLUSION}

In Korea, the age-standardized incidence in age-related CVDs, such as RIA, ICH, and RAVM, were reported at 12.6, 31.3, and 2.4 per 100000 person-years, respectively, in 2016-a declining trend. Meanwhile, UIA and UAVM showed an increasing trend in both crude incidence and age-standardized incidence over 9 years. This trend is likely the result of an increase in the detection rate of CVD and HTN through health screening and preventive surgery and blood pressure control. It may also reflect an increase in the treatment rate due to the development of endovascular treatment and, in particular, an increase in treatment rates in elderly populations. As such, changes in population structure and treatment methods affect the trends in diagnosis and treatment, making these factors particularly critical to our understanding.

\section{CONFLICTS OF INTEREST}

No potential conflict of interest relevant to this article was reported.

\section{INFORMED CONSENT}

This type of study does not require informed consent.

\section{AUTHOR CONTRIBUTIONS}

\author{
Conceptualization : CWO \\ Data curation : SUL, TK \\ Formal analysis : SUL \\ Funding acquisition : $\mathrm{CWO}$ \\ Methodology : SUL \\ Project administration : JSB, OKK \\ Visualization : SPB, HSB \\ Writing - original draft : SUL \\ Writing - review \& editing : SUL, CWO
}

\section{- Acknowledgements}

This study was supported by the Policy Research Funds of the Korean Neurosurgical Society.

\section{References}

1. Adams HP Jr, del Zoppo G, Alberts MJ, Bhatt DL, Brass L, Furlan A, et al. : Guidelines for the early management of adults with ischemic stroke: a guideline from the American Heart Association/American Stroke Association stroke council, clinical cardiology council, cardiovascular radiology and intervention council, and the atherosclerotic peripheral vascular disease and quality of care outcomes in research interdisciplinary working groups: the American academy of neurology affirms the value of this guideline as an educational tool for neurologists. Stroke 38 : 1655-1711, 2007

2. Al-Shahi R, Bhattacharya JJ, Currie DG, Papanastassiou V, Ritchie V, Roberts RC, et al. : Prospective, population-based detection of intracranial vascular malformations in adults: the Scottish Intracranial Vascular Malformation Study (SIVMS). Stroke 34 : 1163-1169, 2003

3. Berman MF, Sciacca RR, Pile-Spellman J, Stapf C, Connolly ES Jr, Mohr JP, et al. : The epidemiology of brain arteriovenous malformations. Neurosurgery 47 : 389-396; discussion 397, 2000

4. Beslow LA, Licht DJ, Smith SE, Storm PB, Heuer GG, Zimmerman RA, et al. : Predictors of outcome in childhood intracerebral hemorrhage: a prospective consecutive cohort study. Stroke 41 : 313-318, 2010

5. Brown RD Jr, Wiebers DO, Torner JC, O'Fallon WM : Incidence and prevalence of intracranial vascular malformations in Olmsted county, Minnesota, 1965 to 1992. Neurology 46 : 949-952, 1996

6. Cheng $\mathrm{CL}$, Chien HC, Lee CH, Lin SJ, Yang YH : Validity of in-hospital mortality data among patients with acute myocardial infarction or stroke in National Health Insurance research database in Taiwan. Int J Cardiol 201 : 96-101, 2015

7. Cheng CL, Kao YH, Lin SJ, Lee CH, Lai ML : Validation of the National Health Insurance research database with ischemic stroke cases in Taiwan. Pharmacoepidemiol Drug Saf 20 : 236-242, 2011 
8. Choi JH, Mohr JP : Brain arteriovenous malformations in adults. Lancet Neurol 4 : 299-308, 2005

9. Ding D, Starke RM, Kano H, Mathieu D, Huang P, Kondziolka D, et al. : Radiosurgery for cerebral arteriovenous malformations in a randomized trial of unruptured brain arteriovenous malformations (ARUBA)-eligible patients: a multicenter study. Stroke 47 : 342-349, 2016

10. Garg K, Singh PK, Sharma BS, Chandra PS, Suri A, Singh M, et al. : Pediatric intracranial aneurysms--our experience and review of literature. Childs Nerv Syst 30 : 873-883, 2014

11. GBD 2016 Causes of Death Collaborators: Global, regional, and national age-sex specific mortality for 264 causes of death, 1980-2016: a systematic analysis for the Global Burden of Disease Study 2016. Lancet 390 : 1151-1210, 2017

12. Hanley DF, Thompson RE, Muschelli J, Rosenblum M, McBee N, Lane K, et al. : Safety and efficacy of minimally invasive surgery plus alteplase in intracerebral haemorrhage evacuation (MISTIE): a randomised, controlled, open-label, phase 2 trial. Lancet Neurol 15 : 1228-1237, 2016

13. Hsieh $\mathrm{CY}$, Chen $\mathrm{CH}$, Li CY, Lai ML : Validating the diagnosis of acute ischemic stroke in a National Health Insurance claims database. J Formos Med Assoc 114 : 254-259, 2015

14. Jauch EC, Saver JL, Adams HP Jr, Bruno A, Connors JJ, Demaerschalk BM, et al. : Guidelines for the early management of patients with acute ischemic stroke: a guideline for healthcare professionals from the American Heart Association/American Stroke Association. Stroke 44 : 870947, 2013

15. Kim H, Sidney S, McCulloch CE, Poon KY, Singh V, Johnston SC, et al. : Racial/Ethnic differences in longitudinal risk of intracranial hemorrhage in brain arteriovenous malformation patients. Stroke 38 : 2430-2437, 2007

16. Koton S, Schneider AL, Rosamond WD, Shahar E, Sang Y, Gottesman RF, et al. : Stroke incidence and mortality trends in US communities, 1987 to 2011. JAMA 312 : 259-268, 2014

17. Krishnamurthi RV, Feigin VL, Forouzanfar MH, Mensah GA, Connor M, Bennett DA, et al. : Global and regional burden of first-ever ischaemic and haemorrhagic stroke during 1990-2010: findings from the Global Burden of Disease Study 2010. Lancet Glob Health 1 : e259-e281, 2013

18. Laakso A, Hernesniemi J : Arteriovenous malformations: epidemiology and clinical presentation. Neurosurg Clin N Am 23 : 1-6, 2012

19. Lackland DT, Roccella EJ, Deutsch AF, Fornage M, George MG, Howard $G$, et al. : Factors influencing the decline in stroke mortality: a statement from the American Heart Association/American Stroke Association. Stroke 45 : 315-353, 2014

20. Lee EJ, Lee HJ, Hyun MK, Choi JE, Kim JH, Lee NR, et al. : Rupture rate for patients with untreated unruptured intracranial aneurysms in South Korea during 2006-2009. J Neurosurg 117 : 53-59, 2012

21. Lee JH, Yang DH, Park HS, Cho Y, Jun JE, Park WH, et al. : Incidence of hypertension in Korea: 5-year follow-up study. J Korean Med Sci 26 : 1286-1292, 2011

22. Liang J, Bao Y, Zhang H, Wrede KH, Zhi X, Li M, et al. : The clinical features and treatment of pediatric intracranial aneurysm. Childs Nerv Syst 25 : 317-324, 2009

23. Magro E, Gentric JC, Batista AL, Kotowski M, Chaalala C, Roberge D, et al. : The Treatment of Brain AVMs Study (TOBAS): an all-inclusive framework to integrate clinical care and research. J Neurosurg 128 : 18231829, 2018

24. Mehrotra A, Nair AP, Das KK, Srivastava A, Sahu RN, Kumar R : Clinical and radiological profiles and outcomes in pediatric patients with intracranial aneurysms. J Neurosurg Pediatr 10 : 340-346, 2012

25. Mendelow AD, Gregson BA, Rowan EN, Murray GD, Gholkar A, Mitchell $P M$, et al. : Early surgery versus initial conservative treatment in patients with spontaneous supratentorial lobar intracerebral haematomas (STICH II): a randomised trial. Lancet 382 : 397-408, 2013

26. Mould WA, Carhuapoma JR, Muschelli J, Lane K, Morgan TC, McBee $N A$, et al. : Minimally invasive surgery plus recombinant tissue-type plasminogen activator for intracerebral hemorrhage evacuation decreases perihematomal edema. Stroke 44 : 627-634, 2013

27. Osbun JW, Reynolds MR, Barrow DL : Arteriovenous malformations: epidemiology, clinical presentation, and diagnostic evaluation. Handb Clin Neurol $143: 25-29,2017$

28. Park JK, Kim KS, Kim CB, Lee TY, Lee KS, Lee DH : The accuracy of ICD codes for cerebrovascular diseases in medical insurance claims. Korean J Prev Med 33 : 76-82, 2000

29. Potts MB, Lau D, Abla AA, Kim H, Young WL, Lawton MT, et al. : Current surgical results with low-grade brain arteriovenous malformations. J Neurosurg 122 : 912-920, 2015

30. Powers WJ, Rabinstein AA, Ackerson T, Adeoye OM, Bambakidis NC, Becker K, et al. : 2018 guidelines for the early management of patients with acute ischemic stroke: a guideline for healthcare professionals from the American Heart Association/American Stroke Association. Stroke 49 : e46-e110, 2018

31. Rothwell PM, Coull AJ, Giles MF, Howard SC, Silver LE, Bull LM, et al. : Change in stroke incidence, mortality, case-fatality, severity, and risk factors in Oxfordshire, UK from 1981 to 2004 (Oxford Vascular Study). Lancet 363 : 1925-1933, 2004

32. Sivenius J, Tuomilehto J, Immonen-Räihä P, Kaarisalo M, Sarti C, Torppa J, et al. : Continuous 15-year decrease in incidence and mortality of stroke in Finland: the FINSTROKE study. Stroke 35 : 420-425, 2004

33. Steiner $T$, Rosand J, Diringer $M$ : Intracerebral hemorrhage associated with oral anticoagulant therapy: current practices and unresolved questions. Stroke 37 : 256-262, 2006

34. Teo M, St George J, Lawton MT : Time for BARBADOS after ARUBA trial. Br J Neurosurg 29 : 635-636, 2015

35. Tonetti DA, Gross BA, Atcheson KM, Jankowitz BT, Kano H, Monaco EA 3rd, et al. : The benefit of radiosurgery for ARUBA-eligible arteriovenous malformations: a practical analysis over an appropriate follow-up period. J Neurosurg 128: 1850-1854, 2018

36. Tsai CF, Thomas B, Sudlow CL : Epidemiology of stroke and its subtypes in Chinese vs white populations: a systematic review. Neurology 81 : 264-272, 2013

37. Wong J, Slomovic A, Ibrahim G, Radovanovic I, Tymianski M : Microsurgery for ARUBA trial (a randomized trial of unruptured brain arteriovenous malformation)-eligible unruptured brain arteriovenous malformations. Stroke 48 : 136-144, 2017 
38. Xia Z, Wu X, Li J, Liu Z, Chen F, Zhang L, et al. : Minimally invasive surgery is superior to conventional craniotomy in patients with spontaneous supratentorial intracerebral hemorrhage: a systematic review and meta-analysis. World Neurosurg 115 : 266-273, 2018
39. Yang W, Caplan JM, Ye X, Wang JY, Braileanu M, Rigamonti D, et al. : Racial associations with hemorrhagic presentation in cerebral arteriovenous malformations. World Neurosurg 84 : 461-469, 2015 